\title{
The Influence of Load and Leg Amputation Upon Coordination in Walking Crustaceans: A Model Calculation
}

\author{
H. Cruse \\ H. Cruse, Universität Bielefeld, Fakultät für Biologie, Bielefeld, Federal Republic of Germany
}

\begin{abstract}
The following results were obtained by earlier authors when investigating the leg coordination of walking crustaceans (Decepoda): 1) After a leg is amputated, its stump moves in anti-phase with the next posterior intact leg. This corresponds to the coordination of intact animals. The stump, however, moves in-phase with the next anterior intact leg which contrasts with the coordination of intact animals (Clarac and Chasserat, 1979; Clarac, 1981). 2) Different results have been reported for the relation between the return stroke duration and step period: some authors found a significant dependency (e.g. MacMillan, 1975), others found none (e.g. Ayers and Davis, 1977). The calculation presented here shows, that these results can be described by a model incorporating the following assumptions: A) The forces developed by both, return stroke and power stroke muscles depend upon the load under which the leg walks. B) The influences which produce the coordinating effects found by Clarac and Chasserat for amputees also exist in intact animals and their strength depends upon the intensity of the motor output of the controlling leg. Within the model the selection of protraction or retraction is made at a "central unit" which calculates a value corresponding to the sum of graded inputs from several sources. The resulting fluctuation in this value might be considered analogous to graded oscillations recorded from central non-spiking interneurons. Qualitatively the model describes similar results obtained from insects.
\end{abstract}

\section{Introduction}

The literature on crustacean walking contains two different hypotheses for describing the mechanism which produces leg coordination (for discussion see Barnes, 1975; Bowerman, 1977; Clarac, 1982; Evoy and Ayers, 1982). One hypothesis, the "alternating tetrapod model" assumes that ipsilateral neighbouring legs always move in anti-phase (phase of 0.5 ) and that both power stroke duration (PS) and return stroke duration (RS) are proportional to step period (which is the sum of PS and RS). This hypothesis may be realized by mutual inhibition between the systems controlling the movement of neighbouring legs (Pearson and Iles, 1973). The second hypothesis assumes that the consecutive steps of the neighbouring ipsilateral legs are controlled by a metachronal wave running from rear to the front. It is further assumed that return stroke duration is constant and only power stroke duration is dependent upon step period. According to this hypothesis the phase between two neighbouring ipsilateral legs can differ from 0.5 and may also depend upon period.

In the literature data have been presented which support each of the hypotheses: A dependence of return stroke duration on period was found in free walking Cardisoma (Evoy and Fourtner, 1973), Uca (Barnes, 1975), Mictyris (Sleinis and Silvey, 1980), and Homarus (McMillan, 1975). In the latter case the animal walked freely on a motor driven treadmill the speed of which was automatically matched to that of the animal. Corresponding results were found for several non crustacean arthropods, including cockroach (Delcomyn, 1971), stick insect (Graham, 1972, Gait I), scorpion (Bowerman, 1975) and wolf spider (Ward and Humphreys, 1981). However, Ayers and Davis (1977) found the return stroke to be independent of period in Homarus tethered on a motor driven treadmill. A similar finding was reported for Cardisoma when walking free but under load (Evoy and Fourtner, 1973).

In the rock lobster a rostro-caudal gradient was evident: the dependence of return stroke duration on period was small for rear legs and increased for more anterior legs (Chasserat and Clarac, 1980; Clarac, 


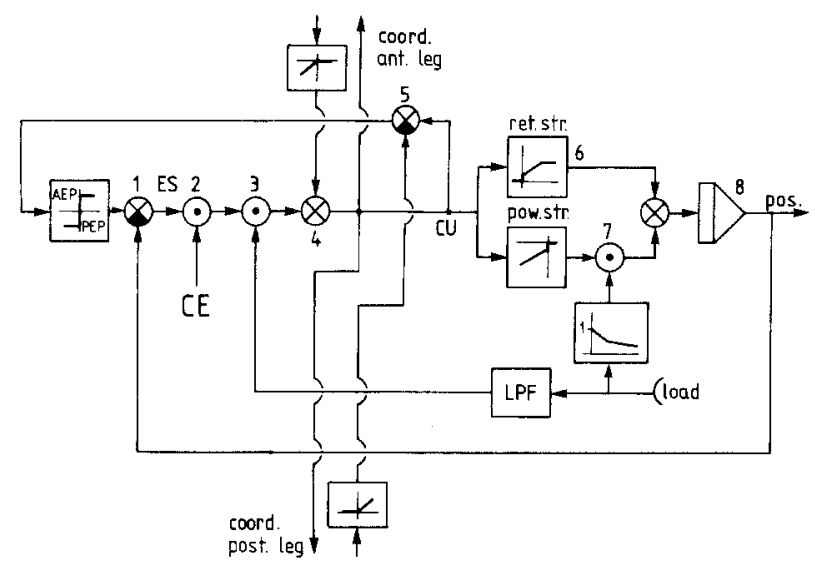

Fig. 1. Wiring diagram of the system describing the movement of a leg. For explanations see text

1981). In insects a constant return stroke duration was found for stick insects when walking on a treadwheel with relatively high inertia (Wendler, 1964) or when walking free (Graham, 1972, Gait II). So the question arises as to why return stroke duration sometimes is dependent upon period and sometimes is not.

Another problem is described as follows: When in the rock lobster one or more neighbouring ispilateral legs are autotomized, the stumps move in-phase with the next anterior intact leg (Clarac and Chasserat, 1979; Clarac, 1981). The experimental results show that this in-phase influence acts in the caudal direction and decreases in strength with increasing distance from the intact leg. These experiments also revealed a weaker anti-phase influence which acts in the forward direction: when several legs are autotomized, the last stump is excited in anti-phase with the next caudal intact leg (Clarac, 1981). This in-phase coupling is not evident in the behaviour of the intact animal and therefore might be interpreted as a special kind of influence only active after autotomy. However, a leg resumes normal coordination when the amputated part of the leg is replaced by an artificial strut [for Homarus: MacMillan (1975), for crayfish: Grote (1981), for stick insect: Wendler (1966)]. Therefore, amputation as such cannot be the cause for the switch to a new type of coupling influence. So the questions arise: Is it possible that the in-phase influence is active in the intact animal although neighbouring legs are walking out of phase? Can a parameter be found to explain the changes in coordination following amputation?

\section{The Model}

Preliminary remarks: The two problems mentioned in the introduction seem to be unrelated at first sight. However, in this paper it is proposed that both groups of experimental results can be explained as effects of the different loads under which the legs walk. On the basis of the results when an amputated leg is replaced by a strut one may conclude that load is an essential parameter for producing a properly coordinated walk. Because the relative durations of power stroke and return stroke change strongly after autotomy of a leg, Clarac (1978) concluded that this relationship may be controlled by some sensory input from the individual leg. It is assumed here that load is this critical sensory information, controlling the relative duration of power and return stroke. Thus both groups of experimental results might be correlated with the parameter load.

It is generally agreed that coupling of legs across the body is less constant than coupling between legs of the same side (e.g. Evoy and Fourtner, 1974; Barnes, 1975; Clarac, 1981). Therefore as a first step in the investigation only coordination of ipsilateral legs will be considered. To avoid unnecessary complication of the description, only forward walking will be treated.

\section{Control of an Individual Leg}

Movement of a walking leg can be considered to be a two state system which is either in a state of return stroke or in power stroke. Thus one needs a central location in the system where the decision will be made which state is active. In the model this decision is performed by the central unit (Fig. 1, CU), a positive output value produces return stroke whereas a negative output value produces power stroke. To produce power and return strokes the central unit is assumed to take on values which alternate rhythmically between positive and negative. Two rectifiers with opposite sign direct the output signal to the appropriate muscles for either the return stroke or the power stroke. [It should be mentioned that apart from the two states "return stroke" and "power stroke" additional states might exist (e.g. "pause", Burns, 1973; MacMillan, 1975; Graham, 1979; "lifting the tarsus", Bässler, 1977). This possibility could be incorporated into the model but for simplicity is not considered here.] The forces developed by these muscles move the leg. The transformation from force to position is approximated by an integrator in the model (Fig. 1, No. 8).

The origin of rhythmicity in the central unit may be produced by a central generator as assumed by several authors. This, however, is not a necessary assumption as shown in the following. Experiments show that the walking rhythm of a leg can be greatly modified by peripheral influences, in particular by preventing a leg from reaching the normal endpoint position of a given phase. When in a rock lobster return stroke movement is interrupted mechanically, the leg stays in this state as long as the leg does not reach its natural anterior 
extreme position (AEP). The same is true for the power stroke and the corresponding posterior extreme position (PEP) (Cruse et al., 1983). Corresponding results are described for insects (Wendler, 1964; Dean and Wendler, 1982; Cruse and Saxler, 1980). This means that there is an input to the central unit which is a) dependent upon the state ("power stroke" or "return stroke") and b) dependent upon position of the leg in such a way that power stroke is finished only when PEP is reached and return stroke only when AEP is reached (further influences as described below might produce some deviations from this rule). Thus one needs a position measuring sense organ the output of which is compared with the reference value PEP during power stroke and AEP during return stroke. In the model a CU producing negative output indicative of power stroke should continue to do so as the difference ("error signal", ES) is negative. As soon as the error signal is zero, the whole system should switch to "return stroke" and take AEP as its reference value. Then the positive error signal should influence the central unit positively to keep on activity of return stroke muscles. Therefore, the simplest hypothesis is to assume that the output of the comparator is an input to the central unit (Fig. 1, ES).

As the reference value is dependent upon the state this value depends upon whether the value of the central unit is positive (which corresponds to return stroke and uses AEP as reference value) or negative (power stroke, PEP, respectively). In the model this is symbolized by a relay characteristic. This simple hypothesis needs no central rhythm generator and corresponds basically to several published models describing leg movements in insects (Wendler, 1968; Graham, 1977; Cruse, 1980). However, the properties of a central rhythm generator can be incorporated in the model; one way in which this can be done is shown below.

The amplitude of the oscillations of the output from the central unit is related to the intensity of muscle forces. As the latter in the intact animal has to be changed by internal commands to produce different walking speeds, in the model the gain of the system can be changed by varying the "central excitation" (Fig. 1, CE, No.2). This assumption corresponds to results obtained from the swimmeret system (Davis, 1971).

As mentioned in the introduction, load is an important parameter in the production of normal leg movement. No details are known about how load changes may influence the control system. Therefore, I want to speculate that increasing load influences the system in the same way as does the central excitation CE, i.e. it increases the gain of the central unit (Fig. 1, No. 3). This assumption is supported by the fact that a leg tied up and unable to contact the ground normally makes only small movements but produces movements of larger amplitudes when the whole animal walks under load (Evoy and Fourtner, 1973; Evoy and Ayers, 1982). Thus an increase in load seems to increase the gain of the whole system. This influence is assumed to be of a tonic nature and is represented by a low pass filter (Fig. 1, LPF) in the model. This means that a higher load increases the gain not only during power stroke but also during return stroke. This agrees with findings in crayfish (Barnes, 1977) and cockroach (Pearson, 1972). For sidewards walking crustaceans Evoy and Fourtner (1973) did not find increased excitation during return stroke with increased load. However, as they could not compare measurements for equal period values in the loaded and unloaded situations, this need not contradict the assumption stated here.

A tonic influence of the load sensitive organs is not the only possible explanation for the increase of motor output during return stroke. Alternatively, the load sensitive organs, which are only phasically stimulated during power stroke, might act additively on a central unit possessing oscillating properties like those of some second order low pass filters. Stronger excitation during power stroke would produce a stronger negative rebound after the end of the power stroke and so increase the excitation during return stroke. Corresponding systems are found in the leech swimming system (Weeks, 1982). Both mechanisms have qualitatively the same effect. I have choosen the first one because the calculation is simpler.

Besides this hypothetical neural influence of increased load a mechanical influence exists which however is present only during power stroke. The greater the load, the slower is the speed of the leg for a given excitation of the power stroke muscles. This is represented in the model by decreasing the gain of the power stroke channel with increasing load (Fig. 1, No. 7). The duration of return stroke reported for different experimental conditions was found to be very variable. However, there seems to be a lower limit. This might be explained by a maximum force value and thus a maximum speed which cannot be exceeded by the return stroke muscles. Therefore for the return stroke muscles a characteristic with saturation is assumed in the model (Fig. 1, No. 6).

\section{Coordination Between Legs}

Clarac and Chasserat (1979) showed that there exists a coordinating influence in the caudal direction which constrains stumps of amputated legs to move in phase with an intact forward leg. This in-phase coordination is opposite to the coordination of intact legs but it is assumed here that this influence nevertheless exists in the intact animal. In addition it is assumed that this 
a

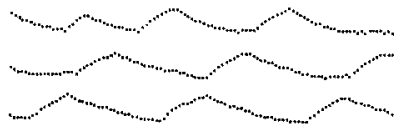

C

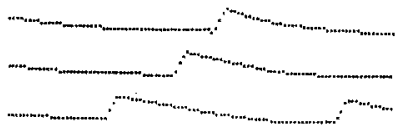

b

$\therefore \therefore \therefore \therefore$

$\because \therefore \therefore \therefore$

$\therefore \quad \therefore \quad \therefore \quad t$

d

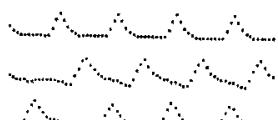

Fig. 2a-d. Coordination of three ipsilateral legs. Upward deflection corresponds to return stroke. Abscissa is time in relative units. Upper trace shows the most anterior leg. The parameters central excitation $C E$ and load $L D$ are varied as follows: a $C E=5, L D=5$; b $C E=40, L D=5 ;$ c $C E=5, L D=50 ; \mathbf{d} C E=40, L D=50$

Table 1. Duration of period (above) and of return stroke (mean values of two steps of every leg) as obtained from the model calculations when varying the parameters central excitation $C E$ and load LD. The time is given in the number of intervals in the time raster illustrated in Figs. 2 and 3

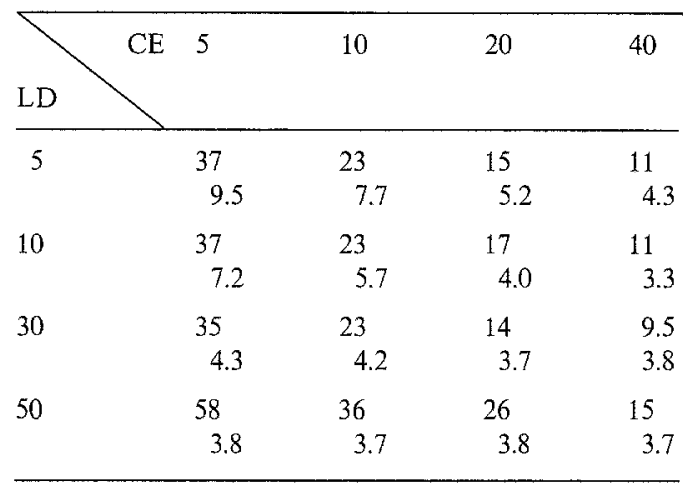

influence works only during power stroke of the anterior leg. (In fact it is sufficient to assume that the influence during power stroke is stronger than during return stroke.) This influence is assumed to work additively on the central unit of the next caudal leg and in this way increases the probability of this leg performing a power stroke when the controlling leg is performing a power stroke (Fig. 1, No. 4). Strong inphase influences between legs were also found in insects and their strength was assumed to depend upon load (Cruse and Saxler, 1980).

Clarac (1981) found another influence directed anteriorly. A leg influences an anterior amputated leg to produce an anti-phase movement. For crustaceans there is no experimental evidence as to how this influence may work. In insects, however, several experimental results (Pearson and Iles, 1973; Graham, 1978; Dean and Wendler, 1982; Cruse and Epstein, 1982) show that during protraction of a leg the probability of the next anterior leg lifting off the ground and starting

a return stroke is diminished. Therefore I assume for crustaceans during the return stroke of the next posterior leg (positive values of CU) a leg's threshold to start a return stroke is made more negative. In the model this is done by a negative influence on the CU value proportional to the $\mathrm{CU}$ value of the posterior leg. This influence is added before the $C U$ value reaches the relay characteristic to produce the position reference input (Fig. 1, No. 5). In the model calculation both, anterior and posterior coordinating influences are weighted by a factor of 0.5 .

Results obtained from insects (Wendler, 1964) and crustacea (Chasserat and Clarac, 1980) show relative coordination which indicates that there exist different eigenfrequencies of the legs with more anterior legs having slightly higher frequencies. Therefore a corresponding hierarchy of eigenfrequencies is assumed in the model.

\section{Results and Discussion}

The model consists of a central unit the output value of which decides whether return stroke or power stroke muscles are activated. The unit receives five inputs. One is proportional to the difference between actual leg position and the position reference value. The second one reflects the changes of central excitation used to produce different force values and different walking speed. The third one depends on the load of the leg. The remaining two describe coordinating influences from other legs: One from the next anterior leg which acts additively on the central unit and influences the leg to walk in phase. The other does not influence the output value of the central unit directly but only changes the threshold value which determines the appropriate position reference value. This influence comes from the next posterior leg and produces antiphase coupling.

The model calculation was restricted to the three ipsilateral legs. It was programmed in BASIC on a Apple microcomputer. The model qualitatively describes coordinated walks at different walking speeds as shown for four examples in Fig. 2. Depending upon the starting positions of the legs occasionally during the first step two neighbouring legs return at the same time, a coordination which occurs sometimes in crustacea, but very rarely in insects. The model does not consider mechanical coupling between the legs. In Table 1 return stroke durations and step periods are given for four different values of "central excitation" CE and of "load" LD. The results show that with this model the return stroke duration is proportional to period when load is small $(\mathrm{LD}=5)$ but does not depend on period when load is high $(\mathrm{LD}=50)$. This occurs because under small loads increasing the central 
a

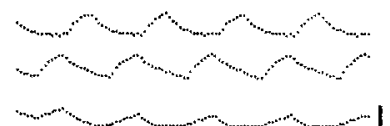

c

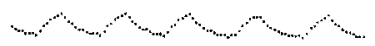

-

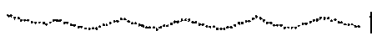

Fig. 3a-d. Coordination of three ipsilateral legs as shown in Fig. 2 with different legs being amputated. $C E=10, L D=5$. The amputated legs are marked by a vertical bar

excitation in order to produce higher walking speeds increases the excitation of the return stroke muscles leading to shorter return strokes. For high load values, however, the saturation level of the return stroke muscles is reached even for small values of central excitation $\mathrm{CE}$. Thus increasing the central excitation does not further decrease the return stroke duration. This agrees with the experimental findings mentioned in the introduction as return stroke seems to be dependent on step period when the animal walks without load and to be dependent on step period when the leg is under load. The model also describes the divergent literature results comparing free walking and walking tethered on a motor driven treadmill. The latter seems to be equivalent to loaded walking. This agrees with the result that in the latter situation the animals develop stronger forces, particularly in slow walks (Clarac and Cruse, 1982). In addition the model shows that simply changing the values of the parameters load and/or central excitation could transform the animals coordination from "metachronal" to "alternating" and it is therefore not necessary that "these two types of gait require fundamentally different forms of inter-leg coordination" as assumed by Evoy and Ayers (1982). The conclusions derived from the model are also supported by the recent finding that return stroke duration is dependent upon load for stick insects (Foth and Graham, 1983).

As Clarac (1981) found a gradient in legs $2-5$ in such a way that the dependency of return stroke duration on period decreased from front to rear, the hypothesis proposed here would predict that the load under which the legs walk in this situation also increases from front to rear. This agrees with experimental results described by Cruse et al. (1983).

One may wonder that power stroke duration increases with load although force increases with load, too. Increased force does not lead to shorter power stroke duration because the model parameters are chosen so that the mechanical influence of the load (Fig. 1, No.7) is stronger than their "neuronal influence (Fig. 1, No. 3).
As shown in Fig. 2 the model produces anti-phase coupling between neighbouring intact legs. This means that the forewards directed influence (Fig. 1, No. 5) has a stronger effect than the backwards directed one (Fig. 1, No. 4). However, when one leg is amputated, which means that this leg is under very small load ( $\mathrm{LD}=0.01$ in the model calculation), then the output value of its central unit is small, i.e. it depends almost solely upon the coordination inputs from neighbouring legs. Conversely, the coordinating influence from an amputated leg on neighbouring legs is small. This means that anti-phase influences continue to dominate between the caudal intact leg and the leg stump, as they do when both legs are intact. However, in-phase coordination occurs between the anterior intact leg and the stump because the normally dominant antiphase influence from the amputated leg is reduced. Therefore the different experimental results found by Clarac are well described by this model as shown in Fig. 3.

The calculation in addition replicates the property found in these experiments that the strength of in phase coupling decreases caudally when several neighbouring legs are amputated. Amputation of the middle leg was also performed with insects (Wendler, 1964). In this situation the system can show relative coordination (Fig. 3d).

Evoy and Fourtner (1973) found that a leg being tied up and therefore unable to touch the ground is moved more strongly when the whole animal walks under load. Therefore the authors state that these influences must be transmitted by neural connections between legs. Similar results were described by Grote (1981) after amputation of a leg. This also is a property of the present model. Increasing the load of one leg increases the output value of its central unit, therefore the amplitude of the coordinating influences are increased as is the value for the central units of neighbouring legs. These influences again are asymmetric and should lead to anti-phase coupling between the two legs which are adjacent to the tied leg. Normally these two legs walk more or less in phase. Both experimental results are also described by the model.

I tried to construct the model with as few elements as possible. Thus several experimental results cannot be described by this simple model. This is mainly due to several interleg and intraleg reflexes which are known to be active in the walking animal (Barnes et al., 1972; Evoy and Fourtner, 1973; Clarac and Coulmance, 1971; Barnes, 1977; Clarac, 1981; Cruse et al., 1983). The main object of this paper is to show that some relatively simple hypotheses collected in this model are able to describe the experimental results mentioned in the introduction. In order to describe the complete known walking behaviour additional con- 
nections might be necessary. One simplification in the model should be mentioned particularly. It is assumed that there exists a definite location where it is decided whether power stroke or return stroke muscles are activated. However, sometimes coactivation of antagonists is observed (e.g. MacMillan, 1975). This might result from a more complex system but, as for some intraleg reflexes, it might also result from additional feedback loops which could easily be fitted to the model without changing its basic properties. This is not described here in detail.

The central unit $\mathrm{CU}$ might be interpreted as an analogue to a pool of non spiking interneurons found in the swimmeret system (Heitler, 1978; Heitler and Pearson, 1980) and the system controlling leg movement of insects (Pearson and Fourtner, 1975). By intracellular current injection these authors were able to reset the rhythmic activity of these interneurons and could therefore show that they were part of the rhythm generator. This experiment works also with the model presented here. By an artificial influence on the value of the central unit the system could be held in the momentary state arbitrarily long or could quickly be switched to the alternative state depending upon the sign (and of course, the amplitude) of this influence. This influence acts in the same way as do the normal coordinating influences.

As mentioned above, there are many experimental results which apply to both, crustaceans and insects. Therefore the model presented here might also be used to describe qualitatively the walking behaviour of insects. However, for insects there is experimental evidence for a caudally directed influence which produces alternating coordination between ipsilateral neighbouring legs (Graham and Baessler, 1981; Bässler, 1983; Bässler and Wegner, 1983). Although such an influence might also exist in crustacea, it has not yet been demonstrated experimentally and therefore is not incorporated in the model calculation presented here.

\section{References}

Ayers, J.L., Davis, W.J.: Neuronal control of locomotion in the lobster, Homarus americanus. I. Motor programs for forward and backward walking. J. Comp. Physiol. 115, 1-27 (1977)

Bässler, U.: Sensory control of leg movement in the stick insect Carausius morosus. Biol. Cybern. 25, 61-72 (1977)

Bässler, U.: Neuralbasis of elementary behaviour in stick insects. Berlin, Heidelberg, New York: Springer 1983

Bässler, U., Wegner, U.: Motor output of the denervated thoracic ventral nerve cord in the stick insect Carausius morosus. J. Exp. Biol. 105, 127-145 (1983)

Barnes, W.J.P.: Leg coordination during walking in the crab, Uca pugnax. J. Comp. Physiol. 96, 237-256 (1975)

Barnes, W.J.P.: Proprioceptive influences on motor output during walking in the crayfish. J. Physiol, (London) 73, 543-564 (1977)
Barnes, W.J., Spirito, C.P., Evoy, W.H.: Nervous control of walking in the crab, Cardisoma guanhumi. II. Role of resistance reflexes in walking. Z. Vergl. Physiol. 76, 16-31 (1972)

Bowermann, R.F.: The control of walking in the scorpion. I. Leg movements during normal walking. J. Comp. Physiol. 100, 183-196 (1975)

Bowermann, R.F.: The contol of arthropod walking. Comp. Biochem. Physiol. 56 A, 231-247 (1977)

Burns, M.D.: The control of walking in Orthoptera. I. Leg movement in normal walking. J. Exp. Biol. 58, 45-58 (1973)

Chasserat, C., Clarac, F.: Interclimb coordinating factors during driven walking in crustacea. J. Comp. Physiol. 139, 293-306 (1980)

Clarac, F.: Locomotory programs in basal leg muscles after limb autotomy in the crustacea. Brain Res. 145, 401-405 (1978)

Clarac, F.: Decapod crustacean leg coordination during walking. In : Locomotion and energetics in arthropods. Herreid, C.F., Fourtner, C.R., (eds), pp. 31-71, New York: Plenum 1981

Clarac, F., Chasserat, C.: Experimental modification of interlimb coordination during locomotion of a crustacea. Neurosci. Lett. 12, 271-276 (1979)

Clarac, F., Coulmance, M.: La marche laterale du crabe (Carcinus). Z. Vergl. Physiol. 73, 408-438 (1971)

Clarac, F., Cruse, H.: Comparison of forces developed by the leg of the rock lobster when walking free or on a treadmill. Biol. Cybern. 43, 109-114 (1982)

Cruse, H.: A quantitative model of walking incorporating central and peripheral influences. I. The control of the individual leg. Biol. Cybern. 37, 131-136 (1980)

Cruse, H., Clarac, F., Chasserat, C.: The control of walking movements in the leg of the rock lobster. Biol. Cybern. 47, 87-94 (1983)

Cruse, H., Epstein, S.: Peripheral influences on the movement of the legs in a walking insect Carausius morosus. J. Exp. Biol. 101, 161-170 (1982)

Cruse, H., Saxler, G.: Oscillations of force in the standing legs of a walking insect Carausius morosus. Biol. Cybern. 36, 159-163 (1980)

Davis, W.J.: Functional significance of motoneurone size and soma position in swimmeret system of the lobster. J. Neurophysiol. 34, 274-288 (1971)

Dean, J., Wendler, G.: Stick insects walking on a wheel: perturbations induced by obstruction of leg protraction. J. Comp. Physiol. 148, 195-207 (1982)

Delcomyn, F.: The locomotion of the cockroach. J. Exp. Biol. 54, 443-452 (1971)

Evoy, W.H., Fourtner, C.R.: Nervous control walking in the crab, Cardisoma guanhumi. III. Proprioceptive influences on intraand intersegmental coordination. J. Comp. Physiol. 83, 303-318 (1973)

Evoy, W., Fourtner, C.R.: Crustacean walking. In: Control of posture and locomotion. Stein, R.B., Pearson, K.B., Smith, R.S., Redford, J.B., (eds), pp. 477-493. New York: Plenum Press 1974

Evoy, W.H., Ayers, J.: Locomotion and control of limb movements. In: The biology of crustacea, D.E. Bliss (ed.), Vol. 4, pp. 61-105. New York, London: Academic Press 1982

Foth, E., Graham, D.: Influence of loading parallel to the body axis on the walking coordination of an insect. Biol. Cybern. 47, 17-23 (1983)

Graham, D.: A behavioural analysis of the temporal organisation of walking movements in the 1 st instar and adult stick insect. $J$. Comp. Physiol. 81, 23-52 (1972)

Graham, D.: Simulation of a model for the coordination of leg movement in free walking insects. Biol. Cybern. 26, 187-198 (1977) 
Graham, D.: Unusual step patterns in the free walking grasshopper, Neoconocephalus robustus. II. A critical test of the leg interactions underlying different models of hexapod $\mathrm{CO}-$ ordination. J. Exp. Biol. 73, 159-172 (1978)

Graham, D.: Effects of circum-oesophageal lesion on the behaviour of the stick insect Carausius. II. Changes in walkingcoordination. Biol. Cybern. 32, 147-152 (1979)

Graham, D., Baessler, U.: Effects of afference sign reversal on motor activity in walking stick insects Carausius morosus. J. Exp. Biol. 91, 179-193 (1981)

Grote, J.R.: The effect of load on locomotion in crayfish. J. Exp. Biol. 92, 277-288 (1981)

Heitler, W.J.: Coupled motoneurons are part of the crayfish swimmeret central oscillator. Nature 275, 231-234 (1978)

Heitler, W.J., Pearson, K.G.: Non-spiking interactions and local interneurons in the central pattern generator of the crayfish swimmeret system. Brain Res. 187, 206-211 (1980)

Macmillan, D.L.: A physiological analysis of walking in the American Lobster Homarus americanus. Philos. Trans. R, Soc. London 270, 1-59 (1975)

Pearson, K.G.: Central programming and reflex control of walking in the cockroach. J. Exp. Biol. 56, 173-193 (1972)

Pearson, K., Fourtner, C.: Nonspiking interneurons in walking system of the cockroach. J. Neurophysiol. 28, 33-52 (1975)

Pearson, K.G., Iles, F.J.: Nervous mechanisms underlying interseg. mental coordination of leg movements during walking in the cockroach. J. Exp. Biol. 58, 725-744 (1973)

Sleinis, S., Silvey, G.E.: Locomotion in a forward walking crab. J. Comp. Physiol. 136, 301-312 (1980)
Ward, T.M., Humphreys, W.F.: Locomotion in burrowing and vagrant wolf spiders (Lycosidae). J. Exp. Biol. 92, 305-321 (1981)

Weeks, J.C.: Synaptic basis of swim initiation in the leech. II. A pattern-generating neuron (cell 208) which mediates motor effects of swim initiating neurons. J. Comp. Physiol. 148, 265-279 (1982)

Wendler, G.: Laufen und Stehen der Stabheuschrecke: Sinnesborsten in den Beingelenken als Glieder von Regelkreisen. Z. Vergl. Physiol. 48, 198-250 (1964)

Wendler, G.: The coordination of walking movements in arthropods. Symp. Soc. Exp. Biol. 20, 229-249 (1966)

Wendler, G.: Ein Analogmodell der Beinbewegungen eines laufenden Insekts. In: Kybernetik 1968, Beihefte zu "elektronischen Anlagen". Vol. 18, pp. 68-74. München, Wien; Oldenbourg 1968

Wendler, G.: Erzeugung und Kontrolle koordinierter Bewegungen bei Tieren-Beispiele an Insekten. In : Kybernetik 1977. Hauske, G., Butenandt, E., (eds.), pp. 11-34. München, Wien; Oldenbourg 1978

Received: August 6, 1983

Holk Cruse

FB Biologie der Universität

Postfach 8640

D-4800 Bielefeld 1

Federal Republic of Germany 